\title{
UTILITY OF RAPID ANTIGEN DETECTION TEST FOR DIAGNOSIS OF STREPTOCOCCAL A INFECTION DIRECTLY FROM THE THROAT SWAB OF SUSPECTED PATIENTS OF PHARYNGITIS
}

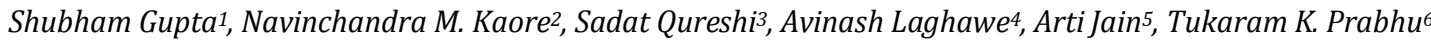 \\ 12nd Year MBBS Student (Professional), PCMS and RC, Bhopal, Madhya Pradesh. \\ ${ }^{2}$ Professor and HOD, Department of Microbiology, RIMS, Raipur, Chhattisgarh, Madhya Pradesh. \\ ${ }^{3}$ Associate Professor, Department of ENT, PCMS and RC, Bhopal, Madhya Pradesh. \\ 4 Professor and HOD, Department of Microbiology, VAMC and RH, Shahajahanpur, Uttar Pradesh. \\ ${ }_{5}^{5}$ Assistant Professor, Department of Microbiology, PCMS and RC, Bhopal, Madhya Pradesh. \\ ${ }^{6}$ Assistant Professor, Department of Microbiology, PCMS and RC, Bhopal, Madhya Pradesh.
}

\begin{abstract}
BACKGROUND
ABSTRACT

Beta-haemolytic Group A Streptococcus (GAS), i.e. Streptococcus pyogenes is responsible for a wide range of both invasive and non-invasive infections. Early diagnosis and treatment of Group A streptococcal pharyngitis has been shown to reduce the severity of symptoms and further complications such as rheumatic fever and glomerulonephritis. Infection of GAS may spread through direct contact with mucus or sores on the skin with over 10 million mild infections and $>500,000$ deaths per year. With $20 \%$ to $40 \%$ bacterial infections, which are indistinguishable from viral infections can be effectively treated by antibiotics. The 48 to 72 hours delay in culture conformation is problematic in low-resource settings, where repeated follow-up visits are not feasible for appropriate treatment. Rapid Antigen Detection Tests (RADTs) assist in the diagnosis of bacterial pharyngitis caused by Group A Streptococci and gives a visual qualitative detection of Group A streptococcal antigen directly from throat swabs. These are potentially more feasible alternatives, because of their quick turnaround time.
\end{abstract}

\section{MATERIALS AND METHODS}

The cross-sectional study was carried out in the Department of Microbiology in college associated with tertiary care hospital, in central India Bhopal during a time period of 2 months from August to September 2016 after obtaining permission from the Institutional Ethics Committee. A total of 38 non-repetitive patients of either gender and of any age clinically suspected to be of pharyngitis attending OPD of Department of ENT in a tertiary care hospital were enrolled after informed consent. Throat swabs collected were transported and processed immediately for the culture and RADT for Streptococcal A. All data was maintained in Microsoft Office Excel and statistical analysis was carried out using Excel with appropriate statistical tools like tests of proportion and significance.

\section{RESULTS}

Out of 38 samples collected, 25 were from male patients and 13 were from females. 35 out of 38 (91.10\%) patients were from active age group. All the samples tested were negative for RADT. A total of 16/38 (42.10\%) samples grew Beta-haemolytic streptococci, but were negative when again tested for Streptococcal A antigen using RADT.

\section{CONCLUSION}

The sensitivity and specificity of Rapid Antigen detection test by immuno-chromatographic tests have been claimed to be over $90 \%$, but could not be established in our settings. A large scale study with strict inclusion criteria is warranted to establish the utility of RADT.

\section{KEYWORDS}

Rapid Antigen Detection Test, Streptococcus Group A, Pharyngeal Swab, RADT, Immuno-Chromatographic Tests.

HOW TO CITE THIS ARTICLE: Gupta S, Kaore NM, Qureshi S, et al. Utility of rapid antigen detection test for diagnosis of streptococcal A infection directly from the throat swab of suspected patients of pharyngitis. J. Evolution Med. Dent. Sci. 2018;7(13): 1616-1619, DOI: $10.14260 /$ jemds/2018/366

\section{BACKGROUND}

Group A Streptococcus (GAS), i.e. Streptococcus pyogenes, the sole species constituting Lancefield group A. This betahaemolytic species of bacteria is responsible for a wide range of both invasive and non-invasive infections. ${ }^{1}$

'Financial or Other Competing Interest': None.

Submission 13-02-2018, Peer Review 10-03-2018,

Acceptance 16-03-2018, Published 26-03-2018.

Corresponding Author:

Dr. Navinchandra M. Kaore,

Professor and HOD, Raipur Institute of

Medical Sciences, Off NH-6, Bhansoj Road,

Godhi, Raipur-492101, Chhattisgarh.

E-mail:navinnmk@gmail.com

DOI: $10.14260 /$ jemds $/ 2018 / 366$

\section{(c) (i) $\$$}

B-haemolytic group A Streptococcus is a major cause of upper respiratory infections such as tonsillitis, pharyngitis, and scarlet fever. Early diagnosis and treatment of Group A streptococcal pharyngitis has been shown to reduce the severity of symptoms and further complications such as rheumatic fever and glomerulonephritis. Rheumatic fever is associated with heart, joint and nervous system damage and serious kidney disease that may result in kidney failure may also be a consequence of streptococcal infection and can be preventable by rapid treatment of streptococcal disease. ${ }^{1}$

Infection of GAS may spread through direct contact with mucus or sores on the skin ${ }^{1}$ and it is estimated that more than 10 million mild infections (throat and skin) occur every year with around $>500,000$ deaths per year. ${ }^{2}$ Majority of pharyngitis is caused by viral infections, but a significant 
proportion i.e. $20 \%$ to $40 \%$ in children and $5 \%$ to $15 \%$ in adults are caused by bacterial infection. ${ }^{3}$ The symptoms of viral and bacterial infection may be indistinguishable, but only bacterial pharyngitis can be effectively treated by antibiotics. Since the major cause of bacterial pharyngitis is GAS, the presence of this organism in a patient's throat may be seen as a necessary condition for prescribing antibiotics. ${ }^{4}$

The gold standard laboratory investigation of GAS pharyngitis is bacterial culture of a throat swab, which require up to 48 to 72 hours for culture, identification and confirmation of Group A Streptococci. Thus, effective management is hindered by the impracticality of throat culture because of the relatively long lag time between the collection of the specimen and final microbiological diagnosis. ${ }^{5}$ This delay is especially problematic in lowresource settings, as it may not be feasible for patients to return for further follow-up visits and appropriate treatment. 6

Rapid Antigen Detection Tests (RADTs) are a potentially more feasible alternative because of their quick turnaround time, so that the clinician can make a decision regarding treatment at the point of care. RADT is a rapid diagnostic test that assist in the diagnosis of bacterial pharyngitis caused by Group A Streptococci and gives a visual, qualitative detection of Group A streptococcal antigen directly from throat swabs.7,8

RADT helps patients both immediately and potentially in the long-term by rapid confirmation of Streptococcal cause of their sore throat. Prompt Antibiotic treatment in confirmed cases provides a quicker reduction of symptoms, shortens the duration of illness, and quickly and efficiently eliminates the possibility of spread of infection to others. It also eliminates the need of antibiotics in Viral Infections, thus preventing the development of antibiotic resistance by not letting unnecessary exposure of antibiotics to commensals.

So this study was planned with an aim to primarily determine the burden of Streptococcal A infection using Rapid Antigen Detection Test in throat swab collected in clinically suspected patients of pharyngitis.

\section{MATERIALS AND METHODS}

This cross-sectional study was carried out in the Department of Microbiology, in college associated with tertiary care hospital in central India Bhopal during a time period of 2 months from August to September 2016 after obtaining permission from the Institutional Ethics Committee. A total of 38 non-repetitive patients of either gender and of any age clinically suspected to be of pharyngitis attending OPD of Department of ENT in a tertiary care hospital were enrolled after informed consent. Two throat swabs were collected with the help of sterile cotton swab moistened with sterile distilled water after depressing the tongue to prevent contamination of the commensal flora and were transported immediately to the microbiology laboratory.

\section{A] Rapid Antigen Detection Test for Streptococcal A}

$1^{\text {st }}$ swab was used for detection of Streptococcal A antigen using SD Strep A from SD Bioline (Standard Diagnostic Inc., Korea 446-930). Manufacturer's instructions were followed, in which 3 drops each of Reagent A and Reagent B were added in the test tube provided. The swab was swirled in the mixture in test tube and allowed to stand for 1 to 2 minutes.
The swab was pressed hard against the wall of the test tube and discarded in $0.5 \%$ sodium hypochlorite solution. The test strip with the arrow facing downwards were placed in the test tube and read at 5 to 10 minutes for the test line to appear along with the control line for validation of the test result.7,8 The positive and negative control run by the controls provided in the kit was also done to increase the validity of the results. The results were interpreted as -1 . Positive test - Test line along with control line, 2. Negative Test - No test line but appearance of control line and 3. Invalid test result - No control line appears.

\section{B] Direct Gram Staining, Culture and Identification}

Second swab was used for culture and preparation of smear on a new grease free glass slide and will be subjected for Gram Staining and findings recorded. 9 The swab was inoculated immediately on Blood Agar and smear was prepared for Direct Examination by Gram staining. Inoculated Blood agar plates were incubated for $18-24 \mathrm{hrs}$. at $37^{\circ} \mathrm{C}$ in capnophilic environment. The Beta-haemolytic colonies on blood agar were further identified as streptococci-A by Gram staining, catalase test and repeat RADT using the colonies on blood agar plate.

All data was maintained in Microsoft Office Excel and Statistical Analysis was carried out using Excel with appropriate statistical tools like tests of proportion and significance.

\section{RESULTS}

The age wise and gender wise distribution of all the 38 samples collected is as given in Table 1.

\begin{tabular}{|c|c|c|c|c|}
\hline \multirow[b]{2}{*}{ Gender } & \multicolumn{3}{|c|}{ Age Group } & \multirow[b]{2}{*}{ Total } \\
\hline & $\begin{array}{c}21 \text { to } 30 \\
\text { Years }\end{array}$ & $\begin{array}{c}31 \text { to } 40 \\
\text { Years }\end{array}$ & $\begin{array}{c}41 \text { to } 50 \\
\text { Years }\end{array}$ & \\
\hline Males & 15 & 08 & 02 & 25 \\
\hline Females & 08 & 04 & 01 & 13 \\
\hline Total & 23 & 12 & 03 & 38 \\
\hline
\end{tabular}

All the 38 samples tested for the Streptococcal-A antigen were found to be negative by RADT. Gram staining and culture results for $2^{\text {nd }}$ throat swab sample processed are as given in Table 2. A total of 16 samples with 8 of either gender were culture positive, but were found to be negative on confirmatory testing with RADT for Streptococcal-A antigen.

\begin{tabular}{|c|c|c|c|}
\hline Gender & \begin{tabular}{|c|} 
Direct Gram \\
Staining \\
showing \\
Gram-Positive \\
Cocci in \\
Chains
\end{tabular} & $\begin{array}{c}\text { Culture } \\
\text { Positive with } \\
\text { Beta } \\
\text { Haemolytic } \\
\text { Colonies }\end{array}$ & $\begin{array}{c}\text { RADT from } \\
\text { Culture } \\
\text { for } \\
\text { Confirmation } \\
\text { of Streptococcal } \\
\text { A }\end{array}$ \\
\hline Males & 05 & 08 & 00 \\
\hline Females & 10 & 08 & 00 \\
\hline Total & 15 & 16 & 00 \\
\hline \multicolumn{4}{|c|}{$\begin{array}{c}\text { Table 2. Direct Gram Staining, Culture Results and RADT } \\
\text { for Confirmation of Streptococcal A by RADT in } 2^{\text {nd }} \text { Throat } \\
\text { Sample Collected }\end{array}$} \\
\hline
\end{tabular}




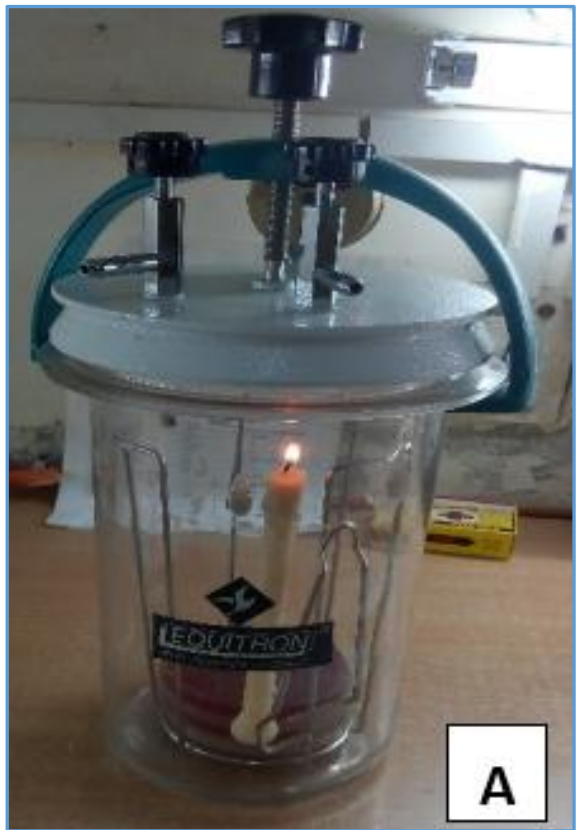

Image 1A. Cultured Plates Incubated in Capnophilic Environment

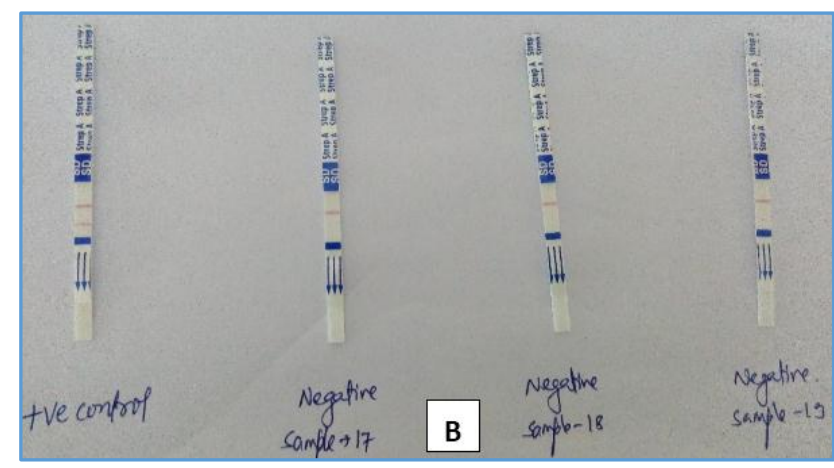

Image 1B. RADT for Streptococcal Antigen with Positive and Negative Control

\section{DISCUSSION}

Streptococcal A infection is the most common bacterial infection causing pharyngitis and accounts for around 5\% to $15 \%$ of the total cases in adults, whereas $20 \%$ to $40 \%$ in children amongst the patients coming with pharyngitis. ${ }^{3}$ Around $35 / 38$ patients $(91.10 \%)$ were from the active age group of 21 to 40 years, in whom the percentage of bacterial pharyngitis is lower. In our study, not a single case of Streptococcal A infection could be detected using the Rapid Antigen Detection Test (RADT).

This is contrary to findings by Ayanruoh $\mathrm{S}$ et al who has shown a positivity of $22.5 \%$ and even from a meta-analytical study, which suggests sensitivity and specificity from $85 \%$ to $95 \%$ for various methods used for antigen detection. ${ }^{10,8}$ The patients catered by our tertiary care hospital are all coming from the lower socioeconomic strata and rural background. Patients are all resorting to the quacks and to the pharmacist for over-the-counter antibiotics and often being given higher antibiotics like fluoroquinolones. The patients reporting after the antibiotic treatment may turn out to be negative for Streptococcal A, as these are all sensitive to used antibiotics. The rampant use of over-the-counter prescriptions, which are quite common in the rural regions of India decrease the positive outcome. 11
Around 15 specimens showed the presence of Gram positive cocci in chains and around 16 specimens showed the growth of Beta-haemolytic colonies. All 15 specimens showing gram positive cocci in chains has shown the growth. None of the streptococci were confirmed as Group A streptococci by repeat RAD test from the growth. This may be because of Beta-haemolytic streptococci other than Group A which might have grown for which RADT used was negative having specificity of over $95 \%$.

None of the sample turns out to be positive by RADT and by culture, may be because of over-the-counter antibiotics or use of antibiotics before visiting the OPD or may be because of the viral aetiology. The sensitivity and specificity cannot be determined nor could the correlation of the RADT with microscopy and the culture be established because of no growth. The less number of samples in our study as compared to various studies conducted remains a limitation because of the cost of the RADT used.

\section{CONCLUSION}

The sensitivity and specificity of Rapid Antigen detection test by immuno-chromatographic tests have been claimed to be over $90 \%$, but could not be established in our settings. A large scale study with strict inclusion criteria is warranted to establish the utility of RADT.

\section{REFERENCES}

[1] Ananthnarayan R, Paniker CKJ. Streptococci. In: Kapil A, edr. Textbook of microbiology. 9th edn. Mumbai: University Press India Ltd., 2013: p. 209-19.

[2] Cohen-Poradosu R, Kasper DL. Group A streptococcus epidemiology and vaccine implications. Clinical Infectious Diseases 2007;45(7):863-5.

[3] Matthys J, De Meyere M, Van Driel ML, et al. Differences among international pharyngitis guidelines: not just academic. Annals of Family Medicine 2007;5(5):436-43.

[4] Danchin MH, Curtis N, Nolan TM, et al. Treatment of sore throat in light of Cochrane verdict: is the jury still out? Medical Journal of Australia 2002;177(9):512-5.

[5] McIsaac WJ, Kellner JD, Aufricht P, et al. Empirical validation of guidelines for management of pharyngitis in children and adults. JAMA 2004;291(13):1587-95.

[6] Rimoin AW, Walker CL, Hamza HS, et al. The utility of rapid antigen detection testing for the diagnosis of streptococcal pharyngitis in low-resource settings. Int J Infect Dis 2010;14(12):e1048-e53.

[7] Gurol Y, Akan H, Izbirak G, et al. The sensitivity and the specifity of rapid antigen test in streptococcal upper respiratory tract infections. Int J Pediatr Otorhinolaryngol 2010;74(6):591-3.

[8] Stewart EH, Davis B, Clemans-Taylor BL, et al. Rapid antigen group A streptococcus test to diagnose pharyngitis: a systematic review and meta-analysis. PLoS One 2014;9(11): e111727.

[9] Collee J, Fraser A, Marmion B, et al. Mackie \& McCartney practical medical microbiology. $14^{\text {th }}$ edn. New Delhi: Churchill Livingstone Imprint of Elsevier 2015;21:387-98. 
[10] Ayanruoh S, Waseem M, Quee F, et al. Impact of rapid streptococcal test on antibiotic use in a pediatric emergency department. Pediatr Emerg Care 2009;25(11):748-50.

10.1097/PEC.0b013e3181bec88c
[11] Llor C, Bjerrum L. Antimicrobial resistance: risk associated with antibiotic overuse and initiatives to reduce the problem. Ther Adv Drug Saf 2014;5(6):229-41. doi: 10.1177/2042098614554919 